\title{
MATERIAL NONLINEAR ANALYSIS OF RIGID PLANE FRAMES BY TOTAL COMPLEMENTARY ENERGY MINIMIZATION
}

\author{
By Sadaji OHKUBO* and Koji MAKINO**
}

\begin{abstract}
A new and powerful material nonlinear analysis method for rigid plane frames is proposed on the basis of the principle of minimum complementary energy and mathematical programming algorithms. The analysis problem is formulated as the total complementary energy minimization problem subjected to the equilibrium equations at the free nodes in terms of the unknown forces acting at the ends of member elements. The unknown member end forces are determined by solving the energy minimization problem using a modified sequential quadratic programming algorithm.

The problem formulation and analysis algorithm of the proposed method are applicable for rigid frame structures with any types of nonlinear materials. The power, reliability, efficiency and practical usefulness of the method presented are demonstrated by comparing the results obtained with ones by the displacement method of analysis for several statically indeterminate rigid frame structures with three types of nonlinear materials.

Keywords: nonlinear analysis, material nonlinearity, rigid plane frame, complementary energy minimization, sequential quadratic programming
\end{abstract}

\section{INTRODUCTION}

Beginning with the early works on limit analysis by Van den Broek ${ }^{1)}$, Prager and Hodge ${ }^{2)}$ and others in the $1940 \sim 50 \mathrm{~s}$, numerous contributions have been devoted to the development of analysis methods for rigid plane frames subjected to material nonlinearity.

The main approaches for the nonlinear frame analysis can be classified into the following two categories : one based on the theory of limit analysis combining linear programming algorithms ${ }^{3)}$ and the other based on the finite element method, in which the stiffness matrix is updated according to the changes in stress state in the finite elements ${ }^{4)}$ ) .

Bogner et al. investigated the formulation of nonlinear frame analysis on the basis of the principle of minimum potential energy with the aid of mathematical programming algorithms in the $1960 \mathrm{~s}^{8)}$.

Recently, Ohkubo et al. developed new analysis methods for trusses and continuous beams based on the energy principles and mathematical programming algorithms. The results are published in Refs.9) 11) . In these studies, the total complementary energy of the structure is expressed in terms of unknown member end forces, axial forces in the truss and bending moments in the continuous girder. The unknown member end forces are determined exactly and effectively by minimizing the total complementary energy subject to equilibrium equations at the free nodes using modified sequential quadratic programming (SQP) and linear programming (LP) algorithms.

In this paper, the analysis methods are extended further, to the rigid plane frame problems subjected to material nonlinearity and a powerful analysis method is proposed.

* Member of JSCE, Dr. Eng., Professor, Department of Civil Engineering, Ehime University (Bunkyo-cho 3, Matsuyama, Ehime)

** Member of JSCE, Kanonji City Office (Kanonjicho kou 300-1, Kanonji, Kagawa) 
In the frame structure, the member elements are each cut at the nodes and the simple span beams system is assumed as a primary structure in which 3 member end forces acting at both ends of each beam are selected as the unknown member end forces. The total complementary energy of a structure and equilibrium equations at the free nodes are expressed in terms of the unknown member end forces, and the unknown forces are determined by minimizing the total complementary energy under equilibrium conditions at the free nodes with the aid of modified SQP and LP algorithms. In the computation of the complementary energy, the contribution of shearing force to it is neglected.

As the numerical examples, the results of portal, 2-bay/1-story and 2-bay/2-story frames with three types of nonlinear materials are demonstrated, and the results are compared with ones obtained by the displacement method of analysis to clarify the generality, accuracy, reliability and efficiency of the proposed method.

\section{TOTAL COMPLEMENTARY ENERGY OF RIGID PLANE FRAME}

\section{(1) Distributions of strain and stress in member cross section by bending moment and axial force}

Consider the stress distribution in a cross section of a beam element which is subjected to a bending moment $M$ and an axial force $N$. If the stress-strain relationship is linear elastic, the stress distribution due to $M$ and $N$ in a beam cross section can be determined simply by adding the stress distributions due to $M$ and $N$. However, if the stress-strain relationship is nonlinear as depicted in Fig. 1, the stress distribution must be determined so as to satisfy the strain distribution and equilibrium conditions in the cross section. In practice, it is recognized as reasonable to assume that plane sections of beam elements which are subjected to a bending moment and an axial force remain plane sections after deformation in the relatively small strain range.

On this assumption, if the magnitudes of extreme fiber strains $\varepsilon_{u}$ and $\varepsilon_{l}$ are assumed, the strain $\varepsilon(y)$ at the point of $y$ from the lower surface of a cross section (see Fig. 2) is given by

$$
\varepsilon(y)=\varepsilon_{l}+\left(\varepsilon_{u}-\varepsilon_{l}\right) / H \cdot y
$$

in which $H$ is the height of the cross section. The stress $\sigma(y)$ corresponding to $\varepsilon(y)$ is given directly from the stress-strain relationship of the material, therefore, $\sigma(y)$ can be expressed as

$$
\sigma(y)=\phi\left(\varepsilon_{u}, \varepsilon_{l}, y\right)
$$

By considering the equilibrium in the cross section, the following equilibrium equations on the normal force and the bending moment are to be satisfied:

$$
\int_{A} \sigma(y) d A=N, \quad-\int_{A} \sigma(y) y d A=M-N y_{\mathrm{G}} \text {. }
$$

in which $y_{G}$ is the distance from the lower surface to the centroid of the cross section.

Substituting Eq. (2) into Eq. (3), we can express the equilibrium equations in terms of $\varepsilon_{u}$ and $\varepsilon_{l}$ : $f_{N}\left(\varepsilon_{u}, \varepsilon_{l}\right)=N, \quad f_{M}\left(\varepsilon_{u}, \varepsilon_{l}\right)=M-N y_{G}$.

The details of $f_{N}$ and $f_{M}$ for a rectangular cross section for the three types of nonlinear materials shown in Fig. 3 are given in the APPENDIX. Eq. (4) is a set of nonlinear simultaneous equations, and by solving the equations for $\varepsilon_{u}$ and $\varepsilon_{l}$, we can determine the strain distribution caused by the given $N$ and $M$. In this paper, the Newton-Raphson method is used to solve Eq. (4). The iterative relation can be written as

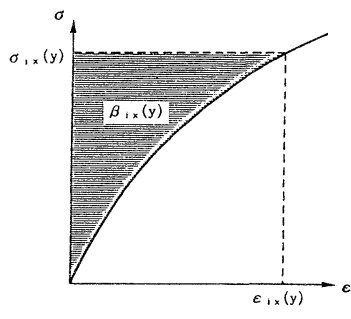

Fig. 1 Nonlinear stress-strain relationship.

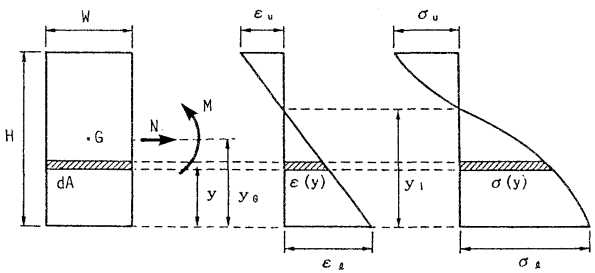

Fig. 2 Strain $(\varepsilon)$ and stress $(\sigma)$ distributions at a finite element. 
$\boldsymbol{r}^{k+1}=\boldsymbol{r}^{k}+\left[\boldsymbol{J}^{k}\right]^{-1} \boldsymbol{S}^{k}$,

where $\quad \boldsymbol{r}^{k}=\left[\varepsilon_{u}^{k}, \varepsilon_{l}^{k}\right]^{T}, \quad \boldsymbol{r}^{\kappa+1}=\left[\varepsilon_{u}^{k+1}, \varepsilon_{l}^{k+1}\right]^{T}$,

$\boldsymbol{S}^{k}=\left[N-f_{N}\left(\boldsymbol{r}^{k}\right), \quad M-N y_{G}-f_{M}\left(\boldsymbol{r}^{k}\right)\right]^{T}$

$\boldsymbol{J}^{K}=\left[\begin{array}{ll}\frac{\partial f_{N}^{k}}{\partial \varepsilon_{u}} & \frac{\partial f_{N}^{k}}{\partial \varepsilon_{l}} \\ \frac{\partial f_{M}^{k}}{\partial \varepsilon_{u}} & \frac{\partial f_{M}^{k}}{\partial \varepsilon_{l}}\end{array}\right]$.

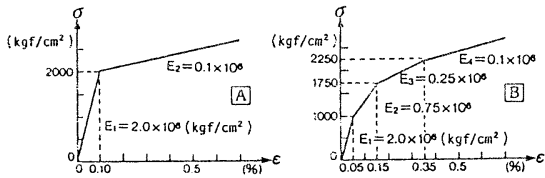

Note that the superscript $T$ denotes the transposed form. Each element in the Jacobian $\boldsymbol{J}$ is calculated approximately by the finite difference formula.

Since the accuracy of $\varepsilon_{u}$ and $\varepsilon_{l}$ calculated by the above process essentially affects the accuracy of the complementary energy in each member element, the convergence criterion in

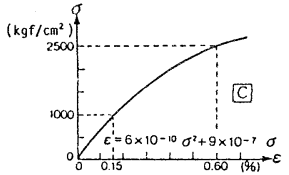

Fig. 3 Three types of nonlinear stress-strain relationships.

the Newton-Raphson process (CNVNWT) is set rigorously as 1/100 of that in the numerical integration of the complementary energy in a member element by the Simpson's rule (CNVSMP). The numerical examples of this investigation on convergence criteria are given in section 5 , but it should be noted here that $5 \sim 10$ iterations of the Newton-Raphson process are sufficient to satisfy the $10^{-5} \sim 10^{-7}$ convergence criterion.

By using $\varepsilon_{u}$ and $\varepsilon_{l}$ obtained by the above process, the strain and then stress distributions in the cross section shown in Fig. 2 can be determined.

(2) Total complementary energy of rigid plane frame

The complementary energy in the cross section per unit length $\pi_{c i}(x)$ at an arbitrary point $x$ in the $i$-th member element is given by

$\pi_{c i}(x)=\int_{A i(x)} \beta_{i x}(y) d A$, where $\beta_{i x}(y)=\int_{0}^{\sigma_{i x}(y)} \varepsilon(\sigma) d \sigma$

$\beta_{i x}(y)$ and $\sigma_{i x}(y)$ are the complementary energy density (the shaded area in Fig. 1) and the stress respectively at the point $y$ from the lower surface in the cross section. $A_{i}(x)$ is the cross-sectional area. The details of calculation formula $\pi_{c i}$ for a rectangular cross section of the three types of nonlinear materials shown in Fig. 3 are also given in the APPENDIX.

The complementary energy in the $i$-th member element with length $l_{i} \Pi_{c i}$ and total complementary energy of the structure with $n$ member elements $\Pi_{c}$ are given by

$$
\Pi_{c i}=\int_{0}^{l_{i}} \pi_{c i}(x) d x, \quad \Pi_{c}=\sum_{i=1}^{n} \Pi_{c i}
$$

In this study, $\Pi_{c i}$ is numerically integrated by using the Simpson's rule. Each member element is partitioned at the points where the cross sections are varied, and concentrated loads are applied. The calculations of $\Pi_{c i}(x)$ in the partitioned section are made at the equally divided points by the $n$-th power of 2. $n$ is started from 1 and is continued to increase until the changing rate in $\Pi_{c i}$ satisfies the imposed convergence criterion CNVSMP $=1.0 \times 10^{-4}$. This convergence criterion CNVSMP is also determined by investigating the accuracy of the final solution due to the magnitude of the criterion. The numerical examples of this investigation are also given in section 5 .

\section{FORMULATION OF ANALYSIS PROBLEM AS COMPLEMENTARY ENERGY MINI- MIZATION PROBLEM}

The principle of minimum complementary energy states that "Among all the sets of admissible stress $\sigma_{x}$, $\sigma_{y}, \cdots \cdots$, and $\tau_{x y}$, which satisfy the equations of equilibrium and the prescribed mechanical boundary conditions on $\boldsymbol{S}_{1}$, the set of actual stress components makes the total complementary energy $\Pi_{c}$ an absolute minimum"12). The part $S_{1}$ over which boundary conditions are prescribed in terms of external forces.

In this study, each member element in a rigid plane frame is released at the nodes, and the simple span 
beams system shown in Fig. 4 (a) is assumed as the primary structure in which the axial and shearing forces and bending moment acting at both ends of each member element (Fig. 4(b)) are the unknown member end forces. However, by using the three equilibrium equations, $\Sigma H=0, \Sigma V=0$, and $\sum M=0$, in the released member element, these six unknown member end forces can be reduced to three unknowns, namely $M_{i j}, M_{i k}$ and $H_{i j}$ in Fig. 4 (c). Using these three unknown forces, the axial force $N_{i}(x)$, bending moment $M_{i}(x)$ and shearing force $S_{i}(x)$ at point $x$ are given as

$$
N_{i}(x)=-H_{i j}+N_{i \rho}(x), \quad M_{i}(x)=\frac{x}{l_{i}}\left(M_{i j}+M_{i k}\right)-M_{i j}+M_{i \rho}(x), \quad S_{i}(x)=\frac{M_{i j}}{l_{i}}+\frac{M_{i k}}{l_{i}}+S_{i \rho}(x) .
$$

In the above expressions, $N_{i p}(x), S_{i p}(x), M_{i p}(x)$ are the axial and shearing forces and bending moment, respectively, acting at point $x$ due to the external loads which are calculated by assuming the released $i$-th member element as a simple beam. Note that the positive sences of $N_{i}, S_{i}$, and $M_{i}$ are in tension, $\uparrow \downarrow$ and $(())$, respectively, in Eq. (9).

As seen from Eq. ( 9$), N_{i}(x), M_{i}(x)$ are expressed in terms of unknown forces $H_{i j}, M_{i j}, M_{i k}$, then the complementary energy in the $i$-th member element $\Pi_{c i}$ and that in the whole structure $\Pi_{c}$ can also be expressed in terms of the unknowns. Namely,

$$
\begin{aligned}
& \Pi_{c i}=\Pi_{c i}\left(H_{i j}, M_{i j}, M_{i \kappa}\right)=\Pi_{c i}\left(X_{i}\right) \cdots \\
& \Pi_{c}=\sum_{i=1}^{n} \Pi_{c i}\left(\boldsymbol{X}_{i}\right)=\Pi_{c}(\boldsymbol{X}), \cdots \\
& \text { where } \quad \boldsymbol{X}=\left[\boldsymbol{X}_{1}^{T}, \boldsymbol{X}_{2}^{T}, \cdots \cdots, \boldsymbol{X}_{n}^{T}\right]^{T} \text {, } \\
& X_{i}=\left[H_{i j}, M_{i j}, M_{i k}\right]^{T}=\left[X_{3 i-2}, X_{3 i-1}, X_{3 i}\right]^{T}
\end{aligned}
$$

Furthermore, the remaining member end forces $V_{i j}, H_{i k}, V_{i k}$, which are to be used for the equilibrium equations at the free nodes, can be expressed as follows.

$$
\left.\begin{array}{l}
V_{i j}=S_{i}(O)=\frac{M_{i j}}{l_{i}}+\frac{M_{i k}}{l_{i}}+S_{i p}(O) \\
H_{i k}=N_{i}\left(l_{i}\right)=-H_{i j}+N_{i p}\left(l_{i}\right) \\
V_{i k}=-S_{i}\left(l_{i}\right)=-\frac{M_{i j}}{l_{i}}-\frac{M_{i k}}{l_{i}}-S_{i p}\left(l_{i}\right)
\end{array}\right)
$$

Then the member end force vector $\boldsymbol{Z}_{i}$ in the $i$-th member element is written in matrix form as $\boldsymbol{Z}_{i}=\boldsymbol{B}_{i}\left[H_{i j}, M_{i j}, M_{i k}\right]^{T}+\boldsymbol{K}_{i}=\boldsymbol{B}_{i} \boldsymbol{X}_{i}+\boldsymbol{K}_{i}$,

$$
\text { where } Z_{i}=\left[H_{i j}, V_{i j}, M_{i j}, H_{i k}, V_{i k}, M_{i k}\right]^{T}
$$

$$
\boldsymbol{B}_{i}=\left[\begin{array}{ccc}
1 & 0 & 0 \\
0 & \frac{1}{l_{i}} & \frac{1}{l_{i}} \\
0 & 1 & 0 \\
-1 & 0 & 0 \\
0 & -\frac{1}{l_{i}} & -\frac{1}{l_{i}} \\
0 & 0 & 1
\end{array}\right], \quad \boldsymbol{K}_{i}=\left[\begin{array}{c}
0 \\
S_{i p}(O) \\
0 \\
N_{i p}\left(l_{i}\right) \\
-S_{i p}\left(l_{i}\right) \\
0
\end{array}\right]
$$

Collecting all of the member end forces $\boldsymbol{Z}_{i}$ in a whole structure, $\boldsymbol{Z}$ is written as

$$
\boldsymbol{Z}=\boldsymbol{B} \boldsymbol{X}+\boldsymbol{K}
$$

$$
\text { where } \boldsymbol{Z}=\left[\boldsymbol{Z}_{1}^{T}, \boldsymbol{Z}_{2}^{T}, \cdots \cdots, \boldsymbol{Z}_{n}^{T}\right]^{T}
$$

$$
\boldsymbol{B}=\left[\begin{array}{ccccc}
\boldsymbol{B}_{1} & & & & 0 \\
& \boldsymbol{B}_{2} & & & \\
& & \cdot & & \\
& & & \cdot & \\
0 & & & & \boldsymbol{B}_{n}
\end{array}\right], \quad \boldsymbol{K}=\left[\begin{array}{c}
\boldsymbol{K}_{1} \\
\cdot \\
\cdot \\
\boldsymbol{K}_{n}
\end{array}\right]
$$

By using Eq. (14), a system of equilibrium equations at the free nodes can be expressed as

$$
F-T Z=F-T K-T B X=0,
$$

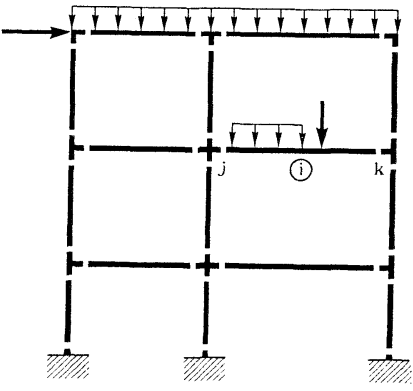

(a)

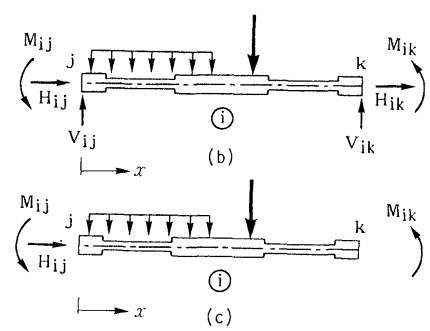

Fig. 4 Primary structure of rigid plane frame, $i$-th member element and three unknown member end forces. 
where $\boldsymbol{T}$ is the force transformation matrix $(m \times 6 n), \boldsymbol{F}$ is the external load vector acting at the free nodes, and $m$ is the degree of freedom of structure. Now let

$\boldsymbol{P}=\boldsymbol{F}-\boldsymbol{T K}, \quad \boldsymbol{C}=\boldsymbol{T B}$.

Then, Eq. (15) can be written as the following simple expression.

$\boldsymbol{P}-\boldsymbol{C X}=\mathbf{0}$

Finally, according to the principle of minimum complementary energy, the problem of determining the true unknown member end forces can be formulated as the following nonlinear complementary energy minimization problem.

$$
\begin{array}{ll}
\text { Find } & \boldsymbol{X} \text {, which } \\
\text { minimize } & \Pi_{c}(\boldsymbol{X})=\sum_{i=1}^{n} \Pi_{c i}\left(\boldsymbol{X}_{i}\right) \\
\text { subject to } & \boldsymbol{g}=\boldsymbol{P}-\boldsymbol{C} \boldsymbol{X}=\mathbf{0}
\end{array}
$$

where $\boldsymbol{g}=\left[g_{1}(\boldsymbol{X}), \cdots \cdots, g_{m}(\boldsymbol{X})\right]^{T}$ are the equilibrium equations at the free nodes.

\section{SOLUTION ALGORITHM}

The total complementary energy minimization problem expressed by Eqs. (18) and (19) is a nonlinear programming problem in which all of the constraints are equality linear constraints and the objective function is a nonlinear function of the unknown member end forces.

Several types of solution algorithms can be applied to solve the minimization problem. However, the sequential quadratic programming (SQP) algorithm ${ }^{14)}{ }^{15)}$, whose efficiency is clarified in Refs.9), 10) and 11), is used as a basis for the solution algorithm. The details of the solution algorithm have been described in Refs.9) and 10), so only the outline is described in this section.

(1) Formulation of approximate quadratic programming problem

By approximating the nonlinear objective function to the quadratic form (Eq. (20)) and expressing the linear equilibrium equations in terms of $\Delta \boldsymbol{X}(\mathrm{Eq}$. (21)), we can introduce the following approximate quadratic programming problem at the $k$-th stage.

Find $\Delta \boldsymbol{X}^{k}$, which

minimize $Q_{c}\left(\Delta \boldsymbol{X}^{k}\right)=\nabla \boldsymbol{\Pi}_{c}^{k T} \Delta \boldsymbol{X}^{k}+\frac{1}{2} \Delta \boldsymbol{X}^{k T} \boldsymbol{H}_{c}^{k} \Delta \boldsymbol{X}^{k}$

subject to $\quad h_{j}\left(\Delta \boldsymbol{X}^{k}\right)=g_{j}\left(\boldsymbol{X}^{k}\right)-\boldsymbol{C}_{j}^{T} \Delta \boldsymbol{X}^{k}=0 \quad(j=1, \cdots \cdots, m) \cdots$

where

$\Delta \boldsymbol{X}^{k}=\left[\Delta \boldsymbol{X}_{1}^{k T}, \cdots \cdots, \Delta \boldsymbol{X}_{n}^{k T}\right]^{T}, \quad \nabla \boldsymbol{\Pi}_{c}^{k}=\left[\frac{\partial \boldsymbol{\Pi}_{c}^{k}}{\partial X_{1}}, \cdots \cdots, \frac{\partial \boldsymbol{\Pi}_{c}^{k}}{\partial X_{3 n}}\right]^{T}$

$H_{c}^{k}$ : positive definite matrix, and improved by the BFGS formula

$\boldsymbol{C}_{j}=\left[C_{j 1}, \cdots \cdots, C_{j 3 n}\right]^{T} \quad(j=1, \cdots \cdots, m)$,

$C_{i j}:(i, j)$ th element of matrix $\boldsymbol{C}(m \times 3 n)$ in Eq. (19).

As clearly seen from Eq. (18) and Fig. 5, only the complementary energy of the $i$-th member element $\Pi_{c i}$ is affected by the changes in unknown member end forces $\Delta X_{3 i-2}, \Delta X_{3 i-1}$, and $\Delta X_{3 i}$. So that the element of $\nabla \Pi_{c}^{k}$, $\partial \Pi_{c}^{k} / \partial X_{3 i-r}(r=2,1,0)$, can be calculated approximately by the following simple finite difference expression. This simplicity in the calculation of sensitivities gives a great computational advantage to the proposed analysis method.

$\partial \Pi_{c}^{k} / \partial X_{3 i-r} \doteqdot\left\{\Pi_{c i}\left(X_{3 i-2}^{k}, X_{3 i-r}^{k}+\Delta X_{3 i-r}, X_{3 i}^{k}\right)-\Pi_{c i}\left(X_{i}^{k}\right)\right\} / \Delta X_{3 i-r}$

\section{( 2 ) Determination of $\Delta \boldsymbol{X}^{k}$}

Since all of the constraints are equality linear constraints, an initial feasible solution $\Delta \boldsymbol{X}_{0}^{k}$ is determined simply by using

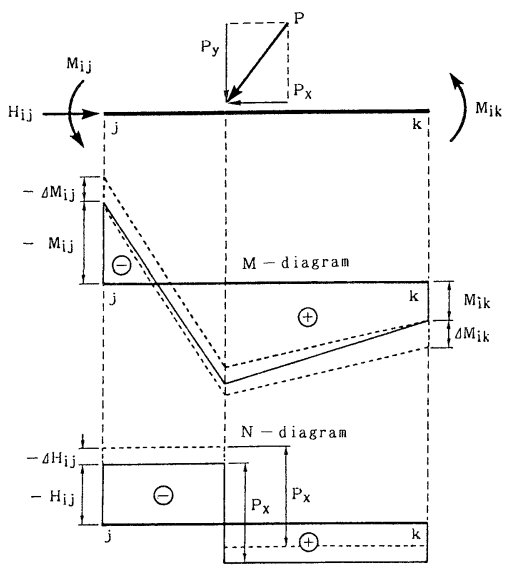

Fig. 5 Changes in $M$ and $N$ distributions due to $\Delta M_{i j}, \Delta M_{i k}, \Delta H_{i j}$. 
the modified linear programming algorithm described in Refs.9) and 10). After the determination of $\Delta \boldsymbol{X}_{0}^{k}$, search direction $\boldsymbol{d}^{k}$ and step length $\alpha^{k}$ to the minimum of $Q_{c}$ along $\boldsymbol{d}^{k}$ are determined by means of the gradient projection method :

$\boldsymbol{d}^{k}=-\operatorname{Pr} \cdot \nabla Q_{c}\left(\Delta X_{0}^{k}\right)$

where $\boldsymbol{P r}=\boldsymbol{I}-\boldsymbol{C}^{T}\left(\boldsymbol{C} \cdot \boldsymbol{C}^{T}\right)^{-1} \boldsymbol{C}, \quad \boldsymbol{I}:$ unit matrix $(3 n \times 3 n)$

$\alpha^{k}=-\left\{\nabla Q_{c}\left(\Delta \boldsymbol{X}_{0}^{k}\right)^{T} \boldsymbol{d}^{k}\right\} / \boldsymbol{d}^{k T} \boldsymbol{H}_{c}^{k} \boldsymbol{d}^{k}$

Then the improvement of $X$ at the $k$-th stage is given by adding $\Delta \boldsymbol{X}_{0}^{k}$ and $\alpha^{k} \boldsymbol{d}^{k}$.

$\Delta \boldsymbol{X}^{k}=\Delta \boldsymbol{X}_{0}^{k}+\alpha^{k} \boldsymbol{d}^{k}$

Because of the equality linear constraints of Eq. (21), although they are expressed in terms of $\Delta \boldsymbol{X}$, are true, i. e. not approximate constraints, once an initial feasible solution $\Delta \boldsymbol{X}_{0}^{k}$ has been determined by the LP algorithm, and if the search direction $\boldsymbol{d}^{k}$ is calculated by Eqs. (23) and (24) without serious round off error at every modification of $\boldsymbol{X}$, the LP algorithm becomes unnecessary.

(3) Modification of approximate quadratic programming problem

By using $\Delta \boldsymbol{X}^{k}$ obtained by $\mathrm{Eq}$. (26), $\boldsymbol{X}^{k+1}$ is improved as

$\boldsymbol{X}^{k+1}=\boldsymbol{X}^{k}+\Delta \boldsymbol{X}^{k}$

Then the values of constraints $g_{j}\left(\boldsymbol{X}^{k+1}\right)(j=1, \cdots \cdots, m)$ and the gradient $\nabla \Pi_{c}$ at $\boldsymbol{X}^{k+1}$ are recalculated. Furthermore the coefficient matrix $H_{c}$ in Eq. (20) is modified by the following BFGS formula :

$$
\boldsymbol{H}_{c}^{k+1}=\boldsymbol{H}_{c}^{k}-\frac{\boldsymbol{H}_{c}^{k} \Delta \boldsymbol{X}^{k} \Delta \boldsymbol{X}^{k T} \boldsymbol{H}_{c}^{k}}{\Delta \boldsymbol{X}^{k T} \boldsymbol{H}_{c}^{k} \Delta \boldsymbol{X}^{k}}+\frac{\eta \eta^{T}}{\Delta \boldsymbol{X}^{k T} \eta}
$$

where $\Delta \boldsymbol{X}^{k}=\boldsymbol{X}^{k+1}-\boldsymbol{X}^{k}, \quad \eta=\nabla \boldsymbol{\Pi}_{c}\left(\boldsymbol{X}^{k+1}\right)-\nabla \boldsymbol{\Pi}_{c}\left(\boldsymbol{X}^{k}\right)$.

The final member end forces $X^{*}$ can be obtained by iterating the improvement of $X$ and the modification of approximate quadratic programming problem stated above until $\Pi_{c}$ and $X$ converge to the constant values. The changing rate $10^{-6}$ is imposed for the convergence criterion on $\Pi_{c}(\mathrm{CNVSQP})$. The details are also given in section 5 .

\section{NUMERICAL EXAMPLES}

The proposed nonlinear analysis method described in sections 2, 3, and 4 has been applied to the analysis models shown in Fig. 6 . The three types of nonlinear materials, the stress-strain relationships are depicted in Fig. 3, are prepared for the frames. The effects of the magnitudes of convergence criteria on the accuracy of final solutions are investigated, and comparisons of the final results obtained by the proposed method and the displacement method of analysis are made to investigate the generality, accuracy, reliability and efficiency of the proposed nonlinear analysis method.

In the proposed analysis method, the initial values of unknown member end forces $X$ are assumed to be of the same sign and same order as those obtained by the linear analysis with the modulus of elasticity $E=2.0$ $\times 10^{6} \mathrm{kgf} / \mathrm{cm}^{2}$, namely \pm 100 ( $\mathrm{tf}$ or $\mathrm{tf} \cdot \mathrm{m}$ ) for all the analysis models.

The material nonlinear analysis of rigid plane frame by the displacement method of analysis has been performed by the following procedures in this study.

(1) Divide each member element into a number of finite beam elements with length $\Delta l$, then assume the axial forces $N$ and bending moments $M$ acting on the ends of each finite beam element. The pseudo axial rigidity $\overline{E A}$ and pseudo flexural rigidity $\overline{E I}$ for each finite beam element are calculated by the following expressions :

$\overline{E A}=N / \varepsilon_{n}$

$\overline{E I}=M \cdot H / \varepsilon_{l}-\varepsilon_{u}$

where $\varepsilon_{l}$ and $\varepsilon_{u}$ are determined by solving the system of equilibrium equations, Eq. (4), substituting assumed $N$ and $M . \varepsilon_{n}$ in Eq. (29) is determined as the corresponding strain to the stress $\sigma_{n}=N / A$ from the stress-strain relationship for the material used. 
(2) Form the pseudo stiffness matrix for the complete structure by assembling the stiffness matrices for each finite beam elements. Then solve the stiffness equations for the unknown displacements and find axial and shearing forces $N, S$ and bending moments $M$ acting at the ends of finite beam elements.

(3) Let $N$ and $M$ computed in (2) be the improved $N$ and $M$ and the procedures (1) and (2) are iterated until all $N, M, \overline{E A}$ and $\overline{E I}$ converge to constant values. The convergence criterion is imposed as $1.0 \times$ $10^{-4}$ to the changing rate in $N, M, \overline{E A}, \overline{E I}$.

In the above displacement method of analysis, the finer partitioning of the finite beam elements yield the more exact solution but requires a much greater core size and computation time. After the comparison of the accuracy of the solutions, core size and computation time required at several values of $\Delta l$ we determined $\Delta l$ to be $10 \mathrm{~cm}$. Moreover, the free nodes were numbered to reduce the band width of structure stiffness matrix mostly to economize on core size and computation time.

(1) Effects of convergence criteria on accuracy of final solutions $X^{*}$ and computation times

In this section, the effects of magnitudes of convergence criteria CNVNWT, CNVSMP, CNVSQP on the accuracy of the final solutions $X^{*}$ and the computation times necessary to obtain $X^{*}$ are investigated.

We have analyzed all the analysis models shown in Fig. 6 for three types of nonlinear materials, A, B and $\mathrm{C}$ depicted in Fig. 3, at various combinations of CNVNWT, CNVSMP and CNVSQP. The numerical results for analysis model 4 , material type $\mathrm{C}$, and comparison with the results by the displacement method of analysis are tabulated in Table 1.

As clearly seen from this table, no relative difference is recognized in the final results for case 1 $\left(\mathrm{CNVSMP}=1.0 \times 10^{-6}\right) \sim 3\left(\mathrm{CNVSMP}=1.0 \times 10^{-4}\right) ;$ however, in case $4\left(\mathrm{CNVSMP}=1.0 \times 10^{-3}\right)$ small relative differences, at most $0.2 \%$, can be observed. The relative differences in the final results for cases $1 \sim 3$ and those obtained by the displacement method of analysis solved with $\Delta l=10 \mathrm{~cm}$ are within the range of $0.04 \sim 1.0 \%$.

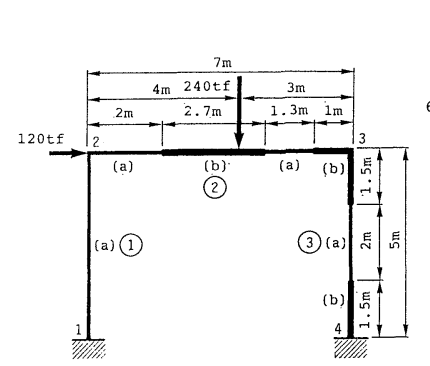

Analysis model 1

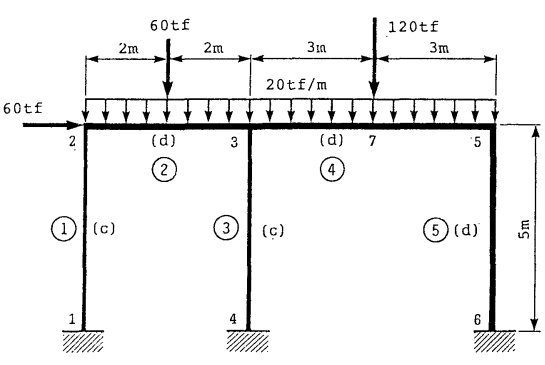

Analysis model 3

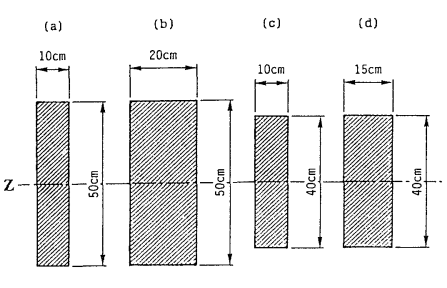

Cross sections

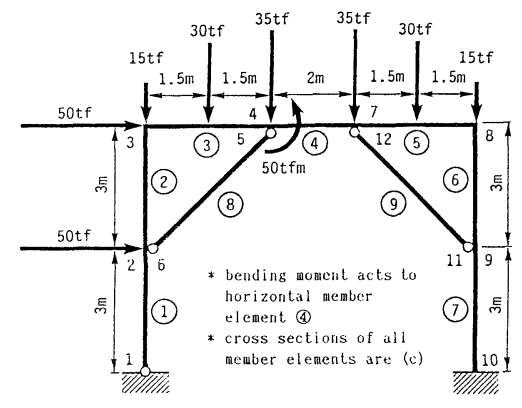

Analysis model. 2

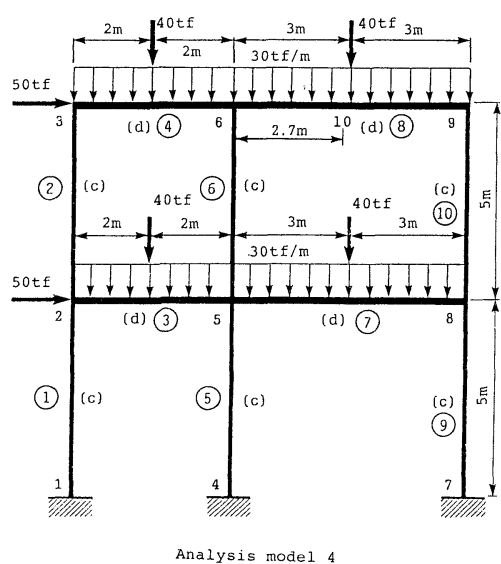

Analysis model 4

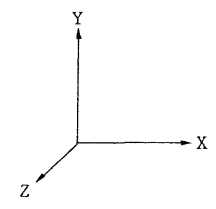

coordinate system

Fig. 6 Analysis models of rigid plane frames. 
Table 1 Comparison of effects of convergence criteria on accuracy of final results and computation times (Analysis model 4, Material type C).

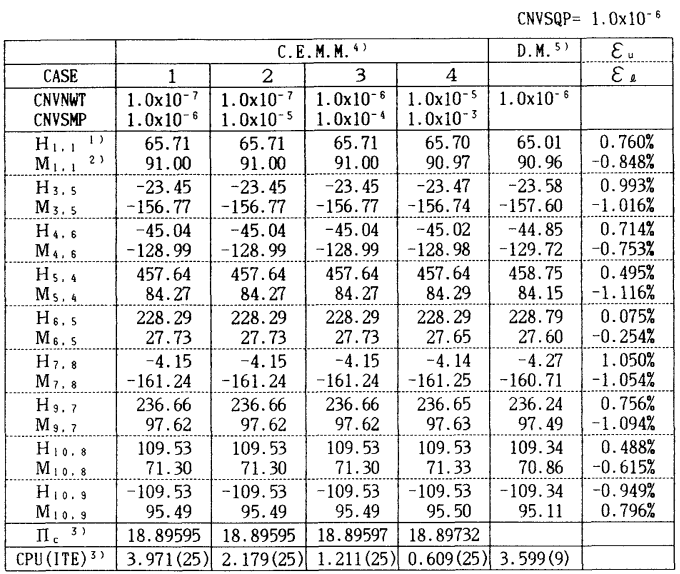

1) Axial force at member end (tf) 2) Bending moment at member end ( $t f \cdot m)$ Computation time by FACOM M-360AP

(number in parenthesis indicate number of iterations)

4) Complementary energy minimization method 5) Displacement method $(1 \mathrm{tf}=9.81 \mathrm{kN})$
Table 2 Comparison of final results by proposed method and displacement method of analysis (Analysis model 2).

\begin{tabular}{|c|c|c|c|c|}
\hline & \multicolumn{4}{|c|}{ Material type $A$} \\
\hline $\begin{array}{l}\text { Analysis } \\
\text { Method }\end{array}$ & C.E.M.M. & D.M. ${ }^{51}$ & r.d. ${ }^{6)}$ & $\varepsilon_{u} / \varepsilon_{\ell}$ \\
\hline $\begin{array}{l}\mathrm{H}_{1,2}{ }^{1} \\
\mathrm{M}_{1,2}{ }^{2}\end{array}$ & & & $\begin{array}{l}0.022 \% \\
0.046 \%\end{array}$ & \\
\hline $\begin{array}{l}\mathrm{H}_{2.2} \\
\mathrm{M}_{2.2}\end{array}$ & & & & \\
\hline $\begin{array}{l}\mathrm{H}_{3,4} \\
\mathrm{M}_{3,4}\end{array}$ & $\begin{array}{r}-94.69 \\
68.42\end{array}$ & & & $\begin{array}{r}-0.172 \% \\
0.136 \%\end{array}$ \\
\hline $\begin{array}{l}\mathrm{H}_{4,7} \\
\mathrm{M}_{4,7}\end{array}$ & $\begin{array}{l}-70.85 \\
-52.56\end{array}$ & $\begin{array}{l}-70.84 \\
-52.53\end{array}$ & & $\begin{array}{r}0.090 \% \\
-0.108 \%\end{array}$ \\
\hline $\begin{array}{l}\mathrm{H}_{6,9} \\
\mathrm{M}_{6,9}\end{array}$ & $\begin{array}{r}-2.75 \\
-96.48 \\
\end{array}$ & $\begin{array}{r}-2.79 \\
-96.50\end{array}$ & $\begin{array}{l}1.434 \% \\
0.021 \%\end{array}$ & $\begin{array}{r}0.781 \% \\
-0.785 \%\end{array}$ \\
\hline $\begin{array}{l}\mathrm{H}_{7.9} \\
\mathrm{M}_{7,9}\end{array}$ & $\begin{array}{r}115.49 \\
96.48\end{array}$ & $\begin{array}{r}115.50 \\
96.50\end{array}$ & $\begin{array}{l}0.009 \% \\
0.021 \%\end{array}$ & $\begin{array}{r}0.728 \% \\
-0.901 \%\end{array}$ \\
\hline $\begin{array}{l}\mathrm{H}_{7.10} \\
\mathrm{M}_{7.10}\end{array}$ & $\begin{array}{r}-115.49 \\
116.08\end{array}$ & $\begin{array}{r}-115.50 \\
116.02\end{array}$ & $\begin{array}{l}0.009 \% \\
0.052 \%\end{array}$ & $\begin{array}{r}-1.656 \% \\
1.399 \%\end{array}$ \\
\hline $\mathrm{H}_{8,5}$ & -33.71 & -33.80 & $0.266 \%$ & $0.004 \%$ \\
\hline & 159.43 & 159.39 & $0.025 \%$ & $-0.020 \%$ \\
\hline $\mathrm{CPU}(\mathrm{ITE})^{3)}$ & $0.707(24)$ & $1.869(29)$ & & \\
\hline
\end{tabular}

1) Axial force at member end(tf)

2) Bending moment at member end $(\mathrm{tf} \cdot \mathrm{m})$

3) Computation time by FACOM M-360AP

(number in parenthesis indicate number of iterations)

4) Compl ementary energy minimization method

5) Displacement method

6) Relative differences of final results by proposed method and displacement method
Table 3 Comparison of final results by proposed method and displacement method of analysis (Analysis model 3).

\begin{tabular}{|c|c|c|c|c|}
\hline & \multicolumn{4}{|c|}{ Material type B } \\
\hline $\begin{array}{l}\text { Analysis } \\
\text { Method }\end{array}$ & C.E.M.M. & D.M. ${ }^{51}$ & r.d. ${ }^{61}$ & $\varepsilon_{u} / \varepsilon_{\ell}$ \\
\hline $\begin{array}{ll}\mathrm{H}_{1,1} & 11 \\
\mathrm{M}_{1,1} & 21\end{array}$ & $\begin{array}{l}30.81 \\
40.29\end{array}$ & $\begin{array}{l}30.87 \\
40.05\end{array}$ & $\begin{array}{l}0.194 \% \\
0.599 \%\end{array}$ & $\begin{array}{r}0.084 \% \\
-0.095 \%\end{array}$ \\
\hline $\begin{array}{l}\mathrm{H}_{2,2} \\
\mathrm{M}_{2,2}\end{array}$ & $\begin{array}{r}46.94 \\
-25.00 \\
\end{array}$ & $\begin{array}{r}47.03 \\
-24.78\end{array}$ & $\begin{array}{l}0.191 \% \\
0.888 \%\end{array}$ & $\begin{array}{r}-0.035 \% \\
0.027 \%\end{array}$ \\
\hline $\begin{array}{l}\mathrm{H}_{2,3} \\
\mathrm{M}_{2.3}\end{array}$ & $\begin{array}{r}-46.94 \\
-131.75\end{array}$ & $\begin{array}{r}-47.03 \\
-131.75\end{array}$ & $\begin{array}{l}0.191 \% \\
0.000 \%\end{array}$ & $\begin{array}{r}0.555 \% \\
-0.591 \% \\
\end{array}$ \\
\hline $\begin{array}{l}\mathrm{H}_{3.3} \\
\mathrm{M}_{3.3}\end{array}$ & $\begin{array}{r}231.57 \\
-6.49\end{array}$ & $\begin{array}{r}231.48 \\
-6.44\end{array}$ & $\begin{array}{l}0.039 \% \\
0.776 \%\end{array}$ & $\begin{array}{l}-0.041 \% \\
-0.017 \%\end{array}$ \\
\hline $\begin{array}{l}\mathrm{H}_{3,4} \\
\mathrm{M}_{3,4}\end{array}$ & $\begin{array}{r}-231.57 \\
23.02 \\
\end{array}$ & $\begin{array}{r}-231.48 \\
23.00\end{array}$ & $\begin{array}{l}0.039 \% \\
0.087 \%\end{array}$ & $\begin{array}{r}-0.084 \% \\
0.019 \%\end{array}$ \\
\hline $\begin{array}{l}\mathrm{H}_{4,3} \\
\mathrm{M}_{4,3}\end{array}$ & $\begin{array}{r}43.64 \\
138.24 \\
\end{array}$ & $\begin{array}{r}43.72 \\
138.19 \\
\end{array}$ & $\begin{array}{l}0.183 \% \\
0.036 \%\end{array}$ & $\begin{array}{r}0.675 \% \\
-0.714 \% \\
\end{array}$ \\
\hline $\begin{array}{l}\mathrm{H}_{5,5} \\
\mathrm{M}_{5,5}\end{array}$ & $\begin{array}{l}117.62 \\
123.97\end{array}$ & $\begin{array}{l}117.66 \\
124.13\end{array}$ & $\begin{array}{l}0.034 \% \\
0.129 \%\end{array}$ & $\begin{array}{r}0.424 \% \\
-0.501 \% \\
\end{array}$ \\
\hline $\begin{array}{l}\mathrm{H}_{5,6} \\
\mathrm{M}_{5,6} \\
\end{array}$ & $\begin{array}{r}-117.62 \\
94.22 \\
\end{array}$ & $\begin{array}{r}-117.66 \\
94.48 \\
\end{array}$ & $\begin{array}{l}0.034 \% \\
0.275 \% \\
\end{array}$ & $\begin{array}{r}-0.231 \% \\
0.188 \% \\
\end{array}$ \\
\hline $\mathrm{CPU}(\mathrm{ITE})^{3)}$ & $1.002(23)$ & $0.579(12$ & & \\
\hline
\end{tabular}

1) Axial force at member end (tf)

2) Bending moment at member end $(t f \cdot m)$

3) Computation time by FACOM M-360AP

(number in parenthesis indicate number of iterations)

4) Complementary energy minimization method

5) Displacement method

6) Relative differences of final results by proposed method and displacement method $\quad(1 \mathrm{tf}=9.81 \mathrm{kN})$
Table 4 Computation time (min.) and number of iterations ${ }^{1)}$

\begin{tabular}{|c|c|c|c|c|c|c|c|c|}
\hline \multicolumn{2}{|c|}{ Analysis Model } & & \multicolumn{2}{|l|}{2} & \multicolumn{2}{|l|}{3} & 4 \\
\hline $\mathrm{n}$ & D.R. ${ }^{21}$ & $3:$ & 3 & 9 & & $5:$ & 6 & $10: 12$ \\
\hline \multirow{2}{*}{$A^{31}$} & C.E.M.M. ${ }^{4 / 51}$ & \multicolumn{2}{|c|}{$0.522(16)$} & \multicolumn{2}{|c|}{$0.707 \quad(24)$} & \multicolumn{2}{|c|}{$0.826(27)$} & $1.903 \quad(31)$ \\
\hline & & \multicolumn{2}{|c|}{$0.208 \quad(13)$} & \multicolumn{2}{|c|}{$1.869(29)$} & \multicolumn{2}{|c|}{$0.669 \quad(15)$} & $* * 40(103)$ \\
\hline & C.E.M.M. & \multicolumn{2}{|c|}{0.565 (15) } & \multicolumn{2}{|c|}{$1.145(22)$} & \multicolumn{2}{|c|}{$1.002 \quad(23)$} & $2.682 \quad(31)$ \\
\hline & D.M. & \multicolumn{2}{|c|}{$0.232(12)$} & \multicolumn{2}{|c|}{$1.022(15)$} & \multicolumn{2}{|c|}{$0.579(12)$} & $6.800(17)$ \\
\hline & C.E.M.M. & \multicolumn{2}{|c|}{0.190 (13) } & \multicolumn{2}{|c|}{$0.329(15)$} & \multicolumn{2}{|c|}{$0.368(15)$} & $1.211 \quad(25)$ \\
\hline & D.M. & 0.147 & (9) & 0.614 & & 0.383 & ( 8 ) & $3.599(9)$ \\
\hline
\end{tabular}

1) Analysis results by FACOM M-360AP 2) Degree of redundancy

3) Material type 4) Complementary energy minimization method

5) Number in parenthesis indicade number of iterations

6) Displacement method

**) did not satisfy the convergence criteria (maximum changing rate is 0.0008 )

Table 5 Examples of displacements in analysis models 2,3 and $4(\Delta l=10 \mathrm{~cm})$

\begin{tabular}{|c|c|c|c|c|}
\hline Analysis Model & Point No. & $\delta_{H}{ }^{1}$ & $\delta_{V}{ }^{2}$ & $\theta$ \\
\hline 2 & 3 & 7.42 & -0.04 & $-0.22 \times 10^{-2}$ \\
\hline \multirow{2}{*}{ Material Type A } & 4 & 7.38 & -0.57 & $0.43 \times 10^{-2}$ \\
\cline { 2 - 5 } & 9 & 7.00 & -0.04 & $-1.61 \times 10^{-2}$ \\
\hline 3 & 2 & 2.28 & -0.02 & $-0.38 \times 10^{-2}$ \\
Material Type B & 3 & 2.26 & -0.14 & $-0.74 \times 10^{-2}$ \\
\hline 4 & 7 & 2.25 & -4.43 & $-0.04 \times 10^{-2}$ \\
\hline \multirow{2}{*}{4} & 3 & 28.68 & -0.13 & $-1.37 \times 10^{-2}$ \\
Material Type C & 5 & 17.86 & -0.91 & $-1.56 \times 10^{-2}$ \\
\cline { 2 - 5 } & 10 & 28.64 & -6.20 & $-0.78 \times 10^{-2}$ \\
\hline
\end{tabular}

1) Horizontal displacement $(\mathrm{cm})$, positive value to the right $(\rightarrow)$ 2) Vertial displacement $(\mathrm{c} m)$, positive value upward $(+)$

3) Rotation(radian), positive to the counterclockwise

The computation time necessary to obtain the final result increases as the values of convergence criteria decrease. Particularly, as the value of CNVSMP decreases, the computation time for $\Pi_{c i}$ increases remarkably and the ratios of computation times for cases 3,2 and 1 to that for case 4 increase to 2. $0,3.6$ and 6.5, respectively. On the other hand, SQP iterations necessary to obtain the final solutions retain the constant values of 25 in all cases.

As a consequence of the above investigation, we decided that the most appropriate combination of the values of CNVNWT and CNVSMP is that in case 3 , in which CNVNWT $=1.0 \times 10^{-6}$ and $C N V S M P=1.0 \times$ $10^{-4}$ for the rigid plane frames such as the analysis models shown in Fig. 6. 
It is noteworthy that the final value of $\Pi_{c}$ is altered slightly in general depending upon the values of CNVNWT and CNVSMP ; however, as seen in Table 1 , the final values of $\boldsymbol{X}, \boldsymbol{X}^{*}$, are not always altered by the values. For the determination of exact $X^{*}$, the significance of the accurate search to the absolute minimum point of $\Pi_{c}$ is to be emphasized in addition to the calculation of $\Pi_{c i}$ with a certain degree of accuracy. For this purpose we investigated the effect of the values of CNVSQP on the accuracy of $X^{*}$ and confirmed that $X^{*}$ could be obtained accurately with the changing rate under $1.0 \times 10^{-4}$ by setting CNVSQP $=1.0 \times 10^{-6}$. Thus we decided upon this value.

\section{(2) Investigation of analysis results}

The analysis models shown in Fig. 6 have been solved by the proposed analysis method (C. E. M. M. ) for the three types of nonlinear materials depicted in Fig. 3, and the results obtained are compared with ones produced by the displacement method of analysis (D. M. ). The numerical results for the analysis model 2 , material type $\mathrm{A}$ and analysis model 3, material type $\mathrm{B}$ are given in Table 2 and 3, respectively, and the computation times and numbers of iterations required to obtain the final solutions by C. E. M. M. for all analysis models in Fig. 6 are summarized in Table 4.

As clearly seen from Tables 1, 2 and 3, the relative differences of the final results by C. E. M. M. and D. M. are within the range of $0.00 \sim 1.00 \%$ in almost all member end forces, as an exception slightly larger differences are observed in the extremely small member end forces. From this fact, it can be said that the final results by both analysis methods are coincide with each other quite accurately. Since the close agreement of the results obtained by D. M. with $\Delta l=10 \mathrm{~cm}$ with the true solutions has been confirmed by comparing the results at various $\Delta l$, it is clear that quite accurate solutions can be determined by the proposed analysis method.

The convergence to the final solutions is excellent. Even if all of the initial values of $\boldsymbol{X}$ are assumed as \pm 100 (tf or $\mathrm{tf} \cdot \mathrm{m})$ for all analysis models, after $4 \sim 10$ iterations, the solutions converge to the neighborhoods of final solutions, and after additional 10 20 iterations the final solutions which satisfy the convergence criteria completely can be determined with certainty. The number of iterations required to obtain the final solutions increases as the degree of redundancy increases; however, the rate of increase is not very high. Moreover, the number of iterations increases with the reduction of the smoothness of nonlinear stress-strain relationships from material type $\mathrm{C}$ to $\mathrm{A}$. However, the computation time increases in the order of C, A, B for all analysis models and is, for example, $0.19 \sim 0.57$ and 1.21 2. 68 minutes for analysis models 1 and 4, respectively, by FACOM-M $360 \mathrm{AP}$.

For references, the computation times and the numbers of iterations necessary to obtain the final solutions by D. M. are also shown in Table 4. In the table, ${ }^{* *}$ at analysis model 4, material type A denotes that the solution did not satisfy the convergence criterion $1.0 \times 10^{-4}$ and the changing rate in $\overline{E A}, \overline{E I}$ still remains at 0.0008 even after 40 minutes of computation and 103 iterations by D. M.

\section{(3) Calculation of displacements at arbitrary points}

Methods of calculating the displacements of structure subjected to the materially nonlinear behavior have been developed in several ways ${ }^{3)-7) .9) .10)}$. An efficient method of calculating the displacements of structure by using complementary energy minimization algorithm developed in this paper has been developed by the author on the basis of the Engesser's first theorem. However, for the limitation of the pages of this paper, the details of the displacement analysis method will be given elsewhere, and we here show only the displacements in the analysis models which were calculated by the following procedures.

(1) By using the final strain distribution in the member elements, which is used for the calculation of the final total complementary energy of the structure, calculate the pseudo axial and flexural rigidities $\overline{E A}$ and $\overline{E I}$ of each finite beam element $(\Delta l=10 \mathrm{~cm})$ by Eqs. (29) and (30).

(2) Assemble the pseudo structure stiffness matrix using $\overline{E A}$ and $\overline{E I}$ of each finite beam element and formulate the stiffness equations for the whole structure. The displacements at arbitrary points are determined by solving the stiffness equations. 
Examples of the numerical values of displacements in analysis models 2, 3 and 4 are given in Table 5. In this table, the positions of noted points are indicated on the analysis models in Fig. 6 by the same numbers.

\section{CONCLUSIONS}

The following conclusions can be drawn from this study.

(1) On the basis of the principle of minimum complementary energy, the materially nonlinear problem of analysis of a rigid plane frame can be formulated as the total complementary energy minimization problem subject to the equilibrium equations at the free nodes in terms of the unknown member end forces. The strain (stress) distribution in a cross section of a beam element which is used for the calculation of complementary energy can be determined readily by solving a system of nonlinear equilibrium equations in the cross section by the Newton-Raphson method. The total complementary energy minimization problem can be solved quite certainly and efficiently by the modified SQP and LP algorithms. The analysis method proposed is a unified approach applicable to rigid plane frame structures with any types of nonlinear materials.

(2) The solution algorithm is quite reliable and efficient. Even if the initial values are assumed to be far away from the final solutions, the solutions enter neighborhoods of the final solutions less than 10 iterations usually. The numbers of iterations to converge to the final solutions increase as the degree of redundancy and the number of member elements increase, but 13 31 iterations have been sufficient for the analysis models shown in Fig. 6 to obtain the final solutions satisfy the strict convergence criteria. The computation times for obtaining final solutions are $0.2 \sim 0.6$ minutes for the portal frame and $1.2 \sim 2.7$ minutes for the 2-bay/2-story frame by FACOM-M $360 \mathrm{AP}$.

( 3 ) The number of unknown variables is three at each member element. Consequently, the analysis problem can be solved with a rather small core size in computation. The improvement of the accuracy of the final solution is accomplished merely by decreasing the values of convergence criteria CNVNWT, CNVSMP and CNVSQP. This small core size requirement and simplicity in accuracy control are great computational advantages of the proposed method.

(4) In all of the analysis models shown in Fig. 6, the accurate final solutions are obtained efficiently by setting $\mathrm{CNVNWT}=1.0 \times 10^{-6}, \mathrm{CNVSMP}=1.0 \times 10^{-4}$, and $\mathrm{CNVSQP}=1.0 \times 10^{-6}$. The relative differences of the final results by the proposed method and the displacement method of analysis are within the range of $0.00 \sim 1.00 \%$ in almost all member end forces; as an exception, slightly larger differences are observed in extremely small member end forces. From this fact, it is clear that quite accurate solutions can be determined by the proposed analysis method.

( 5 ) In the proposed method, the elements of $\nabla \Pi_{c}: \partial \Pi_{c} / \partial X_{3 i-r}(r=2,1,0)$ can be calculated by considering only the changes in $\Pi_{c i}$ with respect to $X_{3 i-r}(r=2,1,0)$. This simplicity also gives a great computational advantage to the proposed analysis method.

\section{ACKNOWLEDGEMENT}

This study was supported in part by the Grant-in-Aid for Scientific Research from the Ministry of Education, Science and Culture of Japan.

\section{APPENDIX Calculation Formulas of $\boldsymbol{f}_{N}, \boldsymbol{f}_{M}$ and $\pi_{c i}$ for Rectangular Section with Height $\boldsymbol{H}$ and Width $W$}

\section{(1) In the cases of material types $A$ and $B$}

$f_{N}=W \sum_{j=1}^{m} A_{j}, \quad f_{M}=-W \sum_{j=1}^{m} A_{j} Y_{G j}, \quad \pi_{c i}=W \sum_{j=1}^{m} \omega_{i j}$,

where $A_{j}, \quad Y_{G j}$ and $\omega_{i j}$ are the area, the distance to centroid from the lower surface and complementary energy of the $j$-th divided area, respectively, in stress distribution (Fig. A.1). Note that $A_{j}$ is positive in 
tension and $\omega_{i j}$ is given by the following processes.

i) $\varepsilon_{k} \geqq 0, \quad \varepsilon_{k+1} \geqq 0$ and $\varepsilon_{k}<0, \quad \varepsilon_{k+1}<0$

The stress at $y_{k} \leqq y \leqq y_{k+1}$ in Fig. $\mathrm{A} \cdot 2$ is expressed as

$\sigma(y)=\sigma_{t}+E_{t}\left(\varepsilon(y)-\varepsilon_{t}\right)$

where $\sigma_{t}, E_{t}, \varepsilon_{t}$ are shown in Fig. A.3. Let $\beta_{t}$ be the complementary energy density at $\sigma_{t}$; then $\beta(y)$ at $\sigma(y)$ is given by

$$
\beta(y)=\beta_{t}+\frac{E_{t}}{2}\left(\varepsilon^{2}(y)-\varepsilon_{t}^{2}\right) \text {. }
$$

By assuming that plane sections through a beam taken normal to its axis remain plane after the beam is subjected to bending moment and axial force, we obtain

$\varepsilon(y)=c y+\varepsilon_{l}, \quad d y=(1 / c) d \varepsilon, \quad c=\left(\varepsilon_{u}-\varepsilon_{l}\right) / H$.

Therefore, $\omega_{i j}$ is

$\omega_{i j}=\int_{y_{k}}^{y_{k+1}} \beta(y) d y=\int_{y_{k}}^{y_{k+1}} \beta_{t} d y+\frac{E_{t}}{2 c} \int_{\varepsilon_{k}}^{\varepsilon_{k+1}}\left(\varepsilon^{2}(y)-\varepsilon_{t}^{2}\right) d \varepsilon=\beta_{t}\left(y_{k+1}-y_{k}\right)+\frac{E_{t}}{c}\left\{\frac{1}{6}\left(\varepsilon_{k+1}^{3}-\varepsilon_{k}^{3}\right)-\frac{\varepsilon_{t}^{2}}{2}\left(\varepsilon_{k+1}-\varepsilon_{k}\right)\right\}$.

(2) In the case of material type $C$

i) $\varepsilon_{u} \geqq 0, \quad \varepsilon_{l} \geqq 0 \quad($ Fig. A.4)

$\varepsilon(y)=a \sigma^{2}(y)+b \sigma(y)$,

where $a=6 \times 10^{-10} \mathrm{~cm}^{4} / \mathrm{kgf}^{2}, \quad b=9 \times 10^{-7} \mathrm{~cm}^{2} / \mathrm{kgf} \quad$ (see Fig. 3).

By using Eqs. $(\mathrm{A} \cdot 4)$ and $(\mathrm{A} \cdot 6)$

$$
y=\frac{a \sigma^{2}(y)+b \sigma(y)-\varepsilon_{l}}{c}, \quad d y=\frac{2 a \sigma(y)+b}{c} \cdot d \sigma .
$$

Therefore, $f_{N}, f_{M}$ and $\pi_{c i}$ can be calculated by the following expressions:

$$
f_{N}=W \int_{0}^{H} \sigma(y) d y=\frac{W}{c} \int_{\sigma_{l}}^{\sigma_{u}} \sigma(y)(2 a \sigma(y)+b) d \sigma=\frac{W}{c}\left\{\frac{2 a}{3}\left(\sigma_{u}^{3}-\sigma_{l}^{3}\right)+\frac{b}{2}\left(\sigma_{u}^{2}-\sigma_{l}^{2}\right)\right\}
$$

in which $\sigma_{u}$ and $\sigma_{l}$ can be obtained by substituting $\varepsilon_{u}$ and $\varepsilon_{l}$ in Eq. (A.6) and solving the equations for $\sigma_{u}$ and $\sigma_{l}$ respectively.

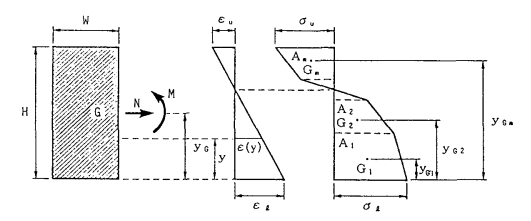

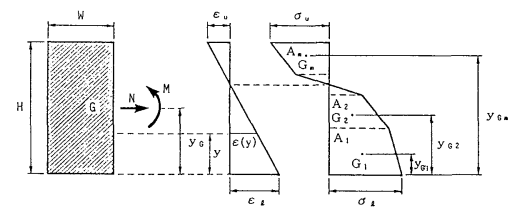$$
f_{M}=-W \int_{0}^{H} \sigma(y) y d y=-\frac{W}{c^{2}} \int_{\sigma_{l}}^{\sigma_{u}} \sigma(y)\left(a \sigma^{2}(y)+b \sigma(y)-\varepsilon_{l}\right)(2 a \sigma(y)+b) d \sigma
$$$$
=\frac{W}{c^{2}}\left\{-\frac{2 a^{2}}{5}\left(\sigma_{u}^{5}-\sigma_{l}^{5}\right)-\frac{3 a b}{4}\left(\sigma_{u}^{4}-\sigma_{l}^{4}\right)-\frac{b^{2}-2 a \varepsilon_{l}}{3}\left(\sigma_{u}^{3}-\sigma_{l}^{3}\right)+\frac{b \varepsilon_{l}}{2}\left(\sigma_{u}^{2}-\sigma_{l}^{2}\right)\right\}
$$$$
\pi_{c i}=W \int_{0}^{H}\left(\int_{0}^{\left.\sigma_{1 y}\right)} \varepsilon(\sigma) d \sigma\right) d y=W \int_{0}^{H}\left(\frac{a}{3} \sigma^{3}(y)+\frac{b}{2} \sigma^{2}(y)\right) d y=\frac{W}{c}\left\{\frac{2 a^{2}}{15}\left(\sigma_{u}^{5}-\sigma_{l}^{5}\right)+\frac{a b}{3}\left(\sigma_{u}^{4}-\sigma_{l}^{4}\right)+\frac{b^{2}}{6}\left(\sigma_{u}^{3}-\sigma_{l}^{3}\right)\right\}
$$

Fig. A. 1 Strain and stress distributions in cross section (Material type A, B).

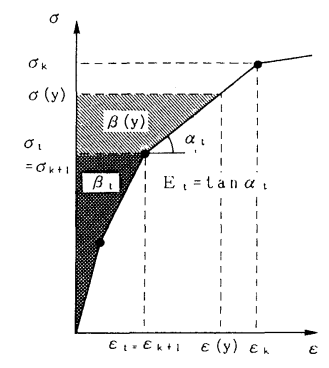

Fig. A.3 A line stress-strain relationship.

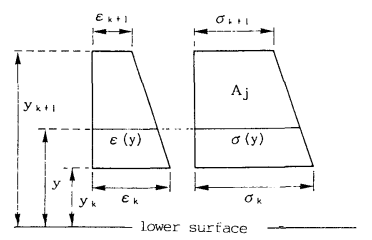

Fig. A.2 Trapezoidal strain and stress distributions in $j$-th divided part.

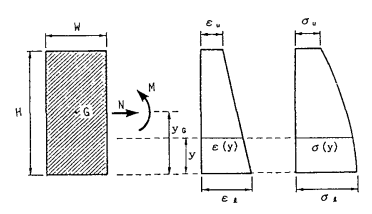

Fig. A.4 Strain and stress distributions in cross section (Material type C). 
ii) $\varepsilon_{u}<0, \quad \varepsilon_{l}>0 \quad$ (see Fig. 2)

If $y_{1}$ is the distance to the point of $\varepsilon=0$ from the lower surface, Eq. (A.7) can be used for $y$ and $d y$ in the range of $\varepsilon \geqq 0$, namely, $0 \leqq y \leqq y_{1}$. In the range of $\varepsilon<0$, namely, $y_{1}<y \leqq H, y$ and $d y$ are given by

$$
y=\frac{-a \sigma^{2}(y)+b \sigma(y)-\varepsilon_{l}}{c}, \quad d y=\frac{-2 a \sigma(y)+b}{c} \cdot d \sigma .
$$

Then, $f_{N}, f_{M}$ and $\pi_{c i}$ are

$$
\begin{aligned}
& f_{N}=W\left(\int_{0}^{y_{1}} \sigma(y) d y+\int_{y_{1}}^{H} \sigma(y) d y\right)=\frac{W}{c}\left\{-\frac{2 a}{3}\left(\sigma_{u}^{3}+\sigma_{l}^{3}\right)+\frac{b}{2}\left(\sigma_{u}^{2}-\sigma_{l}^{2}\right)\right\} \\
& f_{M}=-W\left(\int_{0}^{y_{1}} \sigma(y) y d y+\int_{y_{1}}^{H} \sigma(y) y d y\right) \\
& =\frac{W}{c^{2}}\left\{-\frac{2 a^{2}}{5}\left(\sigma_{u}^{5}-\sigma_{l}^{5}\right)+\frac{3 a b}{4}\left(\sigma_{u}^{4}+\sigma_{l}^{4}\right)-\frac{b^{2}}{3}\left(\sigma_{u}^{3}-\sigma_{l}^{3}\right)-\frac{2 a \varepsilon_{l}}{3}\left(\sigma_{u}^{3}+\sigma_{l}^{3}\right)+\frac{b \varepsilon_{l}}{2}\left(\sigma_{u}^{2}-\sigma_{l}^{2}\right)\right\} \\
& \pi_{c i}=W\left\{\int_{0}^{y_{1}}\left(\frac{a}{3} \sigma^{3}(y)+\frac{b}{2} \sigma^{2}(y)\right) d y+\int_{y_{1}}^{H}\left(-\frac{a}{3} \sigma^{3}(y)+\frac{b}{2} \sigma^{2}(y)\right) d y\right\}=\frac{W}{c}\left\{\frac{2 a^{2}}{15}\left(\sigma_{u}^{5}-\sigma_{l}^{5}\right)-\frac{a b}{3}\left(\sigma_{u}^{4}+\sigma_{l}^{4}\right)+\frac{b^{2}}{6}\left(\sigma_{u}^{3}-\sigma_{l}^{3}\right)\right\} .
\end{aligned}
$$

iii) $\varepsilon_{u}<0, \quad \varepsilon_{l}<0$

In this case, $\varepsilon_{l}, \varepsilon_{u}, \sigma_{l}, \sigma_{u}$ are to be multiplied by -1 first. Then $-f_{N},-f_{M}$ and true $\pi_{c i}$ are calculated by substituting these sign-converted values into Eqs. $(\mathrm{A} \cdot 8),(\mathrm{A} \cdot 9)$ and $(\mathrm{A} \cdot 10)$, respectively. True $f_{N}$ and $f_{M}$ are obtained by converting the signs of $-f_{N}$ and $-f_{M}$.

iv) $\varepsilon_{u}>0, \quad \varepsilon_{l}<0$

In this case, $\varepsilon_{l}, \varepsilon_{u}, \sigma_{l}, \sigma_{u}$ are to be multiplied by -1 first. Then $-f_{N},-f_{M}$ and true $\pi_{c i}$ are calculated by substituting these sign-converted values into Eqs. $(\mathrm{A} \cdot 12),(\mathrm{A} \cdot 13)$ and $(\mathrm{A} \cdot 14)$, respectively. True $f_{N}$ and $f_{M}$ are obtained by converting the signs of $-f_{N}$ and $-f_{M}$.

\section{REFERENCES}

1) Van den Broek, J.A. : Theory of Limit Design, John Wiley and Sons, Inc., New York, 1948.

2) Prager. W. and Hodge, P. G. Jr. : Theory of Perfectly Plastic Solids, John Wiley and Sons, Inc., New York, 1951.

3) Cohn, M. Z. and Maier, G. eds. : Engineering Plasticity by Mathematical Programming, Pergamon Press, New York, 1979.

4) Washizu, K., Miyamoto, H., Yamada, Y., Yamamoto, Y. and Kawai, T. eds. : Handbook of the Finite Element Method-II : Applications, Baihukan, Tokyo, pp.173-269, 1983 (in Japanese).

5) Naruoka, M. Hattori, T., Kato, S., Goto, S. and Ueda, Y. : Analysis of Frame Structures, Lecture Series in Structural Engineering by using Computer II-1-B, JSSC, Baihukan, Tokyo, 1971 (in Japanese).

6) Ohta, T. : Nonelastic Analysis of Structures, New Civil Engineering Series, JSCE ed., Gihodo, Tokyo, pp. 105-122, 1980 (in Japanese).

7) Bathe, K-J. : Finite Element Procedures in Engineering Analysis, Prentice-Hall, Inc., Englewood Cliffs, New Jersey, pp. 301-406, 1982.

8) Bogner, F. K., Mallett, R. H. Minich, M. D. and Schmit, L. A. Jr. : Development and Evaluation of Energy Search Methods of Nonlinear Structural Analysis, Tech. Rep. AFFDL-TR-65-113, Wright-Patterson AFB, Ohio., 1965.

9) Ohkubo, S. and Watada, Y. : Analysis of Trusses with Nonlinear Materials by using Energy Principles and Sequential Quadratic Programming, Proc. of JSCE, No.374/ I-6, pp. 427-436, 1986 (in Japanese)

10) Ohkubo, S. , Watada, Y. and Fujiwaki, T. : Nonlinear Analysis of Truss by Energy Minimization, Computers \& Structures, Vol.27, No. 1, pp. 129-145, 1987.

11) Ohkubo, S. and Fujiwaki, T. : Analysis of Beam Structures with Nonlinear Materials by Total Complementary Energy Minimization, Proc. of JSCE, No.398/ I-10, pp.227-236, 1988 (in Japanese).

12) Washizu, K. : Variational Methods in Elasticity and Plasticity, 3 rd ed., Pergamon Press, Oxford, 1982.

13) Livesley, R. K. : Matrix Methods of Structural Analysis, Pergamon Press, Oxford, chap. 5, 1964.

14) Powell, M. J.D. : A Fast Algorithm for Nonlinearly Constrained Optimization Calculations, Lecture Notes in Mathematics, No. 630, Watson, G. A. ed., Springer, New York, pp. 144-157, 1978.

15) Konno, H. and Yamashita, H. : Nonlinear Programming, Nikkagiren, Tokyo, 1978 (in Japanese).

(Received February 6 1989) 